\title{
A note on generalized characters
}

\author{
R.M. Bryant and L.G. Kovács
}

\begin{abstract}
Let $\alpha$ be a generalized character of a (not necessarily finite) group $G$ over an arbitrary field, and $n$ a positive integer. It is shown that the function $\alpha^{(n)}$ definea by $\alpha^{(n)}(g)=\alpha\left(g^{n}\right)$ is also a generalized character of $G$. An application confirms a conjecture of Robert Higgins and David Ballew: if $G$ is finite and $k$ is also a positive integer, the $k$-th power of the number of $n$-th roots of $g$, summed over all $g$ in $G$, is divisible by the order of $G$.
\end{abstract}

\section{1.}

The representations considered here will all be finite-dimensional linear representations of groups (which may be infinite) over some field $K$. A character will be the character of such a representation and a generalized character the difference of two characters.

If $\alpha$ is a generalized character of a group $G$ and $n$ a positive integer, a function $\alpha^{(n)}$ can be defined by

$$
\alpha^{(n)}(g)=\alpha\left(g^{n}\right)
$$

for all $g \in G$. If $G$ is finite, a second function $\alpha_{(n)}$ can be defined by

$$
\alpha_{(n)}(g)=\sum_{\substack{h \in G \\ h^{n}=g}} \alpha(h),
$$

where $\alpha_{(n)}(g)=0$ if $g$ has no $n$-th root in $G$. We prove 
THEOREM. If $\alpha$ is a generalized character of a group $G$ and $n$ is a positive integer then $\alpha^{(n)}$ is also a generalized character of $G$. If $K$ is the complex field and $G$ is finite then $\alpha_{(n)}$ is also a generalized character of $G$.

In the case where $K$ has characteristic zero, the first part of the theorem is an almost immediate consequence of a well-known formula of Frobenius connecting characters of the general linear group and of the symmetric group (VI (2.3) of [1]), and it may well have been known to Frobenius or his contemporaries. Again, much more general results can probably be found in the context of $K$-theory. However, the theorem in its present elementary form seems interesting enough to warrant explicit statement, and, as far as we know, has not been recorded before.

The second part of the theorem follows from the first, when $G$ is finite and $K$ is the complex field. (One can find examples to show that these conditions cannot be relaxed completely.) The inner product $\langle\alpha, \beta\rangle$ of class functions $\alpha$ and $\beta$ is defined as usual by

$$
\langle\alpha, \beta\rangle=|G|^{-1} \sum_{g \in G} \alpha(g) \beta\left(g^{-1}\right) \text {. }
$$

Thus the class function $\alpha$ is a generalized character of $G$ if and only if $\langle\alpha, \beta\rangle$ is integral for every generalized character $\beta$ of $G$. If $\alpha$ and $\beta$ are generalized characters, one checks easily that

$$
\langle\alpha(n), \beta\rangle=\left\langle\alpha, \beta^{(n)}\right\rangle \text {. }
$$

Since $\beta^{(n)}$ is a generalized character of $G$, by the first part of the theorem, $\alpha_{(n)}$ is also a generalized character of $G$, and this establishes the second part.

If $\rho$ is the trivial character of a finite group $G$, then $\rho_{(n)}(g)$ is the number of $n$-th roots of $g$ in $G$. By the theorem, $\rho_{(n)}$, and consequently any power $\left(\rho_{(n)}\right)^{k}$, is a generalized character of $G$ (over the complex field). This verifies the conjecture in [3] which states in effect that $\left\langle\left(\rho_{(n)}\right)^{k}, \rho\right\rangle$ is an integer. 
2.

To prove the first part of the theorem it is sufficient to consider the case where $\alpha$ is a faithful character of $G$. Therefore, since a generalized character remains a generalized character upon restriction, we may take $G$ to be the whole general linear group, on some finite-dimensional vector space $V$, and $\alpha$ the trace function on $G$. In this section, $G, V$ and $\alpha$ have these meanings and $n$ is a positive integer. $S_{n}$ will denote the symmetric group on $\{1, \ldots, n\}$.

Firstly we note that $K$ is a splitting field for $S_{n}$ and that every character of $S_{n}$ is integer-valued: it is well-known that the rationals form a splitting-field for $S_{n}$ (\$129 of [5]), and so, by Corollary 83.7 of [2] for example, every prime field is a splitting field for $S_{n}$. Secondly, every irreducible $K\left(G \times S_{n}\right)$-module is the outer tensor product (p. 315 of [2]) of an irreducible $K G$-module and an irreducible $K S_{n}$-module. This is because Corollary 51.13 of [2] holds, without any finiteness condition on the groups, for any field which is a splitting field for at least one of the groups. The proof in [2] may be adapted to this case. It follows that if $\zeta$ is an irreducible character of $G \times S_{n}$ then there are characters $\eta$ of $G$ and $\xi$ of $S_{n}$ such that, for all $g \in G, h \in S_{n}$, we have $\zeta(g h)=n(g) \xi(h)$.

Let $W$ be the $n$-fold tensor power of $V$, and for $v_{1}, \ldots, v_{n} \in V$, $g \in G$, and $h \in S_{n}$, define

$$
\left(v_{1} \otimes \ldots \otimes v_{n}\right) g=v_{1} g \otimes \ldots \otimes v_{n} g,
$$

and

$$
\left(v_{1} \otimes \ldots \otimes v_{n}\right) h=v_{(1) h^{-1}} \otimes \ldots \otimes v_{(n) h^{-1}}
$$

This extends to a definition of $W$ as a $K\left(G \times S_{n}\right)$-module (as in 567 of [2]), affording a representation of $G \times S_{n}$ with character $\zeta$, say. If we choose a basis for $V$ and a corresponding basis for $W$ an easy 
calculation shows that, for $g \in G, h \in S_{n}$,

$$
\zeta(g h)=\alpha(g)^{h(1)} \ldots \alpha\left(g^{n}\right)^{h(n)},
$$

where $h(i)$ is the number of cycles of $h$ of length $i$. On the other hand $\zeta=\Sigma \zeta_{i}$ is a finite sum of irreducible characters of $G \times S_{n}$. Thus, by the remark above, there are characters $\eta_{i}$ of $G$ and $\xi_{i}$ of $S_{n}$ such that

$$
\zeta(g h)=\Sigma n_{i}(g) \xi_{i}(h)
$$

If in (1) and (2) we take $h$ to be a cycle of order $n$, we find

$$
\alpha^{(n)}=\Sigma \xi_{i}(h) n_{i}
$$

As remarked above, the $\xi_{i}(h)$ are integers, and so $\alpha^{(n)}$ is a generalized character of $G$.

If in (1) and (2) an element $g$ of $G$ is fixed, there results an expression for a class function of $S_{n}$ : this is the basis of one method of determining the irreducible characters of $S_{n}$ (VI, \$3 of [1]). By suitable choice of $g$ one obtains generalized characters of $S_{n}$. In particular, if $K$ is the field of the rationals, and $g$ is taken to be a permutation matrix, one gets the generalized characters of Theorem 5.1 of [4], obtained there in a rather different way.

REMARK (added in proof, 11 June 1971). Dr Ballew has informed us that he and Mr Higgins jointly, and Professor Richard Braver independently, have also confirmed the conjecture of [3].

\section{References}

[1] Hermann Boerner, Representations of groups with special consideration for the needs of moderm physics (translated by P.G. Murphy in cooperation with $\mathrm{J}$. Mayer-Kalkschmidt and P. Care. North-Holland, Amsterdam; Interscience [John Wiley \& Sons], New York, 1963). 
[2] Charles W. Curtis; Irving Reiner, Representation theory of finite groups and associative algebras (Pure and Applied Mathematics, XI. Interscience [John Wiley \& Sons], New York, London, 1962).

[3] Robert Higgins and David Ballew, "An equation for finite groups", Amer. Math. Monthly 78 (1971), 274-275.

[4] A. Rudvalis and E. Snapper, "Numerical polynomials for arbitrary characters", J. Combinatorial Theory Ser. A 10 (1971), 145-159.

[5] B.L. van der Waerden, Modern algebra, VoZ. II (translated from the second revised German edition by Theodore J. Benac. Frederick Ungar, New York, 1950).

Institute of Advanced Studies, Australian National University, Canberra, ACT. 